\title{
SUPERNOVA ABUNDANCE GENERATION
}

\author{
K. NOMOTO, T. SHIGEYAMA, and T. TSUJIMOTO \\ Department of Astronomy, Faculty of Science, University of Tokyo, Tokyo 113
}

\begin{abstract}
Theoretical models of supernova explosions of various types are reviewed to obtain heavy element yields from supernovae. We focus on new models for SN 1987A, and Type Ia, Ib, and Ic supernovae. Maximum brightness and decline rate of their light curves suggest that $12-18 M_{\odot}$ stars produce larger amount of ${ }^{56} \mathrm{Ni}$ than more massive stars. We discuss relative roles of various types of supernovae in the chemical evolution of galaxies.
\end{abstract}

\section{SUPERNOVA PROGENITORS AND SUPERNOVA TYPES}

Supernovae have been classified into several spectroscopically distinct types (e.g., Harkness and Wheeler 1990). Supernovae of Type I (SNe I) and Type II (SNe II) are respectively defined by the absence and presence of hydrogen in their optical spectra. Classical SNe Ia are characterized by the presence of a strong Si II line. $\mathrm{SNe} \mathrm{Ib}$ and Ic, by contrast, do not show this strong line (see, however, $\S 3$ for $\mathrm{SNe}$ Ic). Moderately strong $\mathrm{He}$ I lines distinguish $\mathrm{SNe} \mathrm{Ib}$ from $\mathrm{SNe}$ Ic at early times.

The late-time optical spectra of SNe provide additional constraints on the classification scheme. SNe Ia show strong blends of Fe emission lines. SNe Ib and Ic, on the other hand, are dominated by emission lines of intermediate-mass elements such as $\mathrm{O}$ and $\mathrm{Ca}$. SNe II are dominated by the strong $\mathrm{H} \alpha$ emission line.

The light curves of most SNe I are similar and very likely to be powered by the decays of ${ }^{56} \mathrm{Ni}$ and ${ }^{56} \mathrm{Co}$, although some variations in the initial rate of decline have been noted. Well-observed SNe Ib are about 1.5 mag fainter than SNe Ia at maximum (see, however, $\S 3$ for SNe Ic).

Large differences have been known in light curves of SNe II. A subdivision of $\mathrm{SNe}$ II into two distinct subclasses has been proposed. SNe II-P ("plateau") have light curves that decline only a small amount during the first 2 months after maximum (plateau). The light curves of SNe II-L ("linear"), by contrast, decline without showing a plateau.

Figure 1 summarizes the currently proposed initial masses $M_{\mathrm{i}}$ of the progenitors for the various types of supernovae. The upper and lower rows respectively show 


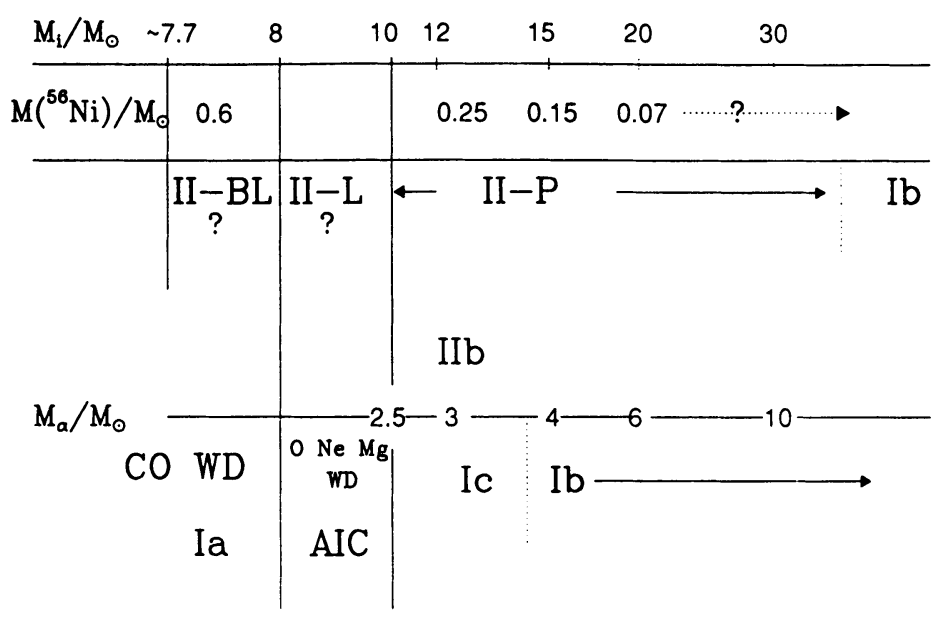

Fig. 1: Hypothetical connection between supernova types and their progenitors for single stars (upper) and close binary stars (lower). $M_{\mathrm{i}}$ and $M_{\alpha}$ are the initial mass and the helium star mass, respectively. AIC stands for accretion-induced collapse of white dwarfs.

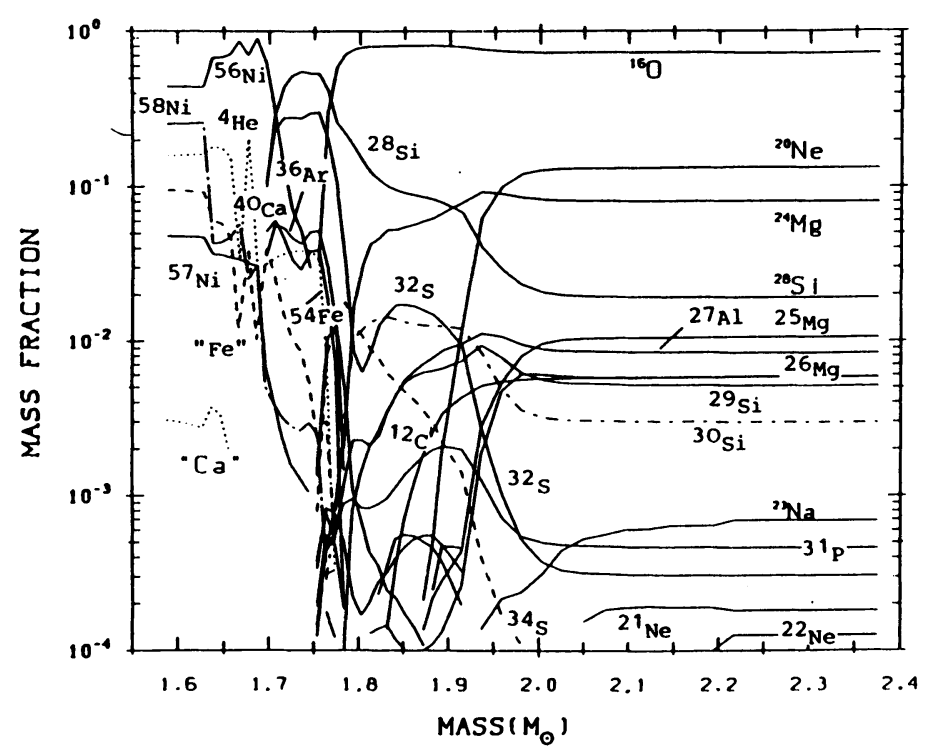

Fig. 2: Abundance distribution after passage of the supernova shock front in SN 1987A (Hashimoto et al. 1989). Matter outside $2 M_{\odot}$ is essentially unaltered. Mass zones further in experience explosive $\mathrm{Si}, \mathrm{O}, \mathrm{Ne}$, and $\mathrm{C}$-burning. 
the cases of single stars and helium stars of masses $M_{\alpha}$ (or white dwarfs) in close binary stars. The produced masses of ${ }^{56} \mathrm{Ni}$ inferred from light curves are also given. Among these types of supernovae, we focus on new models for SN 1987A, SNe Ia, $\mathrm{Ib}$, and Ic, and discuss their relative roles in the chemical evolution of galaxies.

\section{EXPLOSIVE NUCLEOSYNTHESIS IN SN 1987A}

SN 1987A in the LMC has provided us with an excellent materials to test the theory of massive star evolution, nucleosynthesis, and supernova explosion. Its progenitor has been identified with a blue supergiant star Sk-69 202 . From its luminosity $\sim 1.3 \times 10^{5} L_{\odot}$, the helium core mass of the progenitor is estimated as $M_{\alpha} \sim 6 M_{\odot}$ whose initial mass was $M_{\mathrm{i}} \sim 20 \pm 2 M_{\odot}$ (Arnett et al. 1989 for a review).

Here we summarize the nucleosynthesis products from the theoretical model of SN 1987A and compare with the observations. The initial model for the explosion calculation are constructed from the pre-collapse model of $6 M_{\odot}$ helium core and the hydrogen-rich envelope. The model named 14E1 consists of a point mass neutron star of $1.6 M_{\odot}$, a heavy element layer of $2.2 M_{\odot}$, a helium-rich layer of $2.2 M_{\odot}$, and a hydrogen-rich envelope of $10.2 M_{\odot}$. The final kinetic energy of explosion is assumed to be $E=1 \times 10^{51}$ ergs (Shigeyama et al. 1988; Shigeyama and Nomoto 1990).

\subsection{Explosive Nuclear Burning}

As the shock wave propagates through the Si and O-rich layers, explosive nucleosynthesis takes place behind the shock (Hashimoto et al. 1989; Thielemann et al. 1990). Nucleosynthesis is determined by the peak temperature $T$ as follows:

(1) Explosive Silicon Burning: Temperatures in excess of $5 \times 10^{9} \mathrm{~K}$ lead to complete Si-exhaustion and produce only Fe-group nuclei. The most abundant nucleus is ${ }^{56} \mathrm{Ni}$ for the neutron excess less than $2 \times 10^{-2}$ or $Y_{\mathrm{e}}>0.49$. Other less abundant nuclei are ${ }^{57} \mathrm{Ni}$ and ${ }^{58} \mathrm{Ni}$ as well as trace abundances of ${ }^{40} \mathrm{Ca},{ }^{44} \mathrm{Ti},{ }^{48} \mathrm{Cr}$, and ${ }^{52} \mathrm{Fe}$.

Incomplete Si-burning is characterized by peak temperatures of $4-5 \times 10^{9} \mathrm{~K}$. Besides the dominant fuel nuclei ${ }^{28} \mathrm{Si}$ and ${ }^{32} \mathrm{~S}$, the alpha-nuclei ${ }^{36} \mathrm{Ar}$ and ${ }^{40} \mathrm{Ca}$ are most abundant. Also small amounts of the Fe-group (mostly ${ }^{56} \mathrm{Ni}$ and ${ }^{54} \mathrm{Fe}$ and some ${ }^{52} \mathrm{Fe},{ }^{58} \mathrm{Ni},{ }^{55} \mathrm{Co}$, and ${ }^{57} \mathrm{Ni}$ ) are produced.

(2) Explosive Oxygen Burning: Temperatures in excess of $\sim 3.3 \times 10^{9} \mathrm{~K}$ lead to a quasi-equilibrium among nuclei in the range $28<A<45$ in mass number. The main burning products are ${ }^{28} \mathrm{Si},{ }^{32} \mathrm{~S},{ }^{36} \mathrm{Ar},{ }^{40} \mathrm{Ca},{ }^{38} \mathrm{Ar}$, and ${ }^{34} \mathrm{~S}$. Also ${ }^{33} \mathrm{~S},{ }^{39} \mathrm{~K}$, ${ }^{35} \mathrm{Cl},{ }^{42} \mathrm{Ca}$, and ${ }^{37} \mathrm{Ar}$ show up with mass fractions less than $10^{-2}$. In zones with temperatures close to $4 \times 10^{9} \mathrm{~K}$ there exists still a contamination by the Fe-group nuclei ${ }^{54} \mathrm{Fe},{ }^{56} \mathrm{Ni},{ }^{52} \mathrm{Fe},{ }^{58} \mathrm{Ni},{ }^{55} \mathrm{Co}$, and ${ }^{57} \mathrm{Ni}$.

(3) Explosive Neon and Carbon Burning: The main burning products are ${ }^{16} \mathrm{O},{ }^{24} \mathrm{Mg}$, and ${ }^{28} \mathrm{Si}$, synthesized via the reaction sequences ${ }^{20} \mathrm{Ne}(\gamma, \alpha){ }^{16} \mathrm{O}$ and ${ }^{20} \mathrm{Ne}$ 
$(\alpha, \gamma){ }^{24} \mathrm{Mg}(\alpha, \gamma){ }^{28} \mathrm{Si}$, similar to the hydrostatic case. Zones with peak temperatures in excess of $2.1 \times 10^{9} \mathrm{~K}$ undergo a combined version of explosive Ne and C-burning. Besides the major abundances mentioned above, Ne-burning supplies also substantial amounts of ${ }^{27} \mathrm{Al},{ }^{29} \mathrm{Si},{ }^{32} \mathrm{~S},{ }^{30} \mathrm{Si}$, and ${ }^{31} \mathrm{P}, \mathrm{C}$-burning contributes in addition the nuclei ${ }^{20} \mathrm{Ne},{ }^{23} \mathrm{Na},{ }^{24} \mathrm{Mg},{ }^{25} \mathrm{Mg}$, and ${ }^{26} \mathrm{Mg}$.

In the $20 M_{\odot}$ star, the maximum temperatures $T$ behind the shock are shown in Hashimoto et al. (1989), where $T$ is approximately given by $E=4 \pi r^{3} / 3 a T^{4}$ for the sphere of radius $r$ (e.g., Woosley 1988). The region with $T>5 \times 10^{9} \mathrm{~K}$, for example, corresponds to a sphere of radius $\sim 3700\left(E / 10^{51} \mathrm{erg}\right)^{1 / 3} \mathrm{~km}$ which contains $1.7 M_{\odot}$.

Explosive nucleosynthesis for $E=1 \times 10^{51}$ erg takes place as follows:

(1) complete Si-burning extends out to $1.69 M_{\odot}$,

(2) incomplete Si-burning starts at $1.69 M_{\odot}$ and extends out to $1.74 M_{\odot}$,

(3) explosive O-burning occurs up to $1.8 M_{\odot}$, and

(4) explosive Ne-burning leads to an ${ }^{16} \mathrm{O}$-enhancement over its hydrostatic value in the mass zones up to $2 M_{\odot}$.

The resulting abundance distribution is shown in Fig. 2. The integrated abundances of stable isotopes relative to the solar values (normalized to ${ }^{28} \mathrm{Si}$ ) are shown in Hashimoto et al. (1989).

\subsection{The Light Curve and ${ }^{56} \mathrm{Ni}$ Mass}

What can we learn about nucleosynthesis from the photometric and spectroscopic observations of SN 1987A, especially, from its quite unique optical light curve (Fig. $3)$ ? The early light curve due to shock heating was $~ 20$ times dimmer than that of a typical SNe II-P because of the smaller radius of the blue-superginat progenitor. As a result, the later light curve has provided unique information on nucleosynthesis in the interior (Shigeyama et al. 1988; Woosley 1988; Arnett 1988).

After day $\sim 25$, the theoretical model predicts that the light curve is powered by radioactive decays as follows (Fig. 4). When a ${ }^{56} \mathrm{Co}$ decays to a ${ }^{56} \mathrm{Fe}, \gamma$-ray photons are emitted with the energy of $847 \mathrm{keV}, 1028 \mathrm{keV}, 1238 \mathrm{keV}$, etc.. The average energy of photons emitted by ${ }^{56} \mathrm{Co}$ is about $1.28 \mathrm{MeV}$ and the number of these photons is 2.88 on average. These $\gamma$-ray photons are degraded into $\mathrm{X}$-rays by Compton scatterings and absorbed by heavy elements (e.g., Kumagai et al. 1989). In the first several scatterings, an electron takes about a half of the photon energy per scattering. These high energy electrons collide with ions or atoms to ionize or excite them. The ionized electrons are thermalized through the same processes. In this way, the energy of $\gamma$-rays emitted by the ${ }^{56} \mathrm{Co}$ decay is deposited in the supernova ejecta and powers the optical light.

After a plateau-like broad peak, the observed luminosity declined slowly for $t=$ 


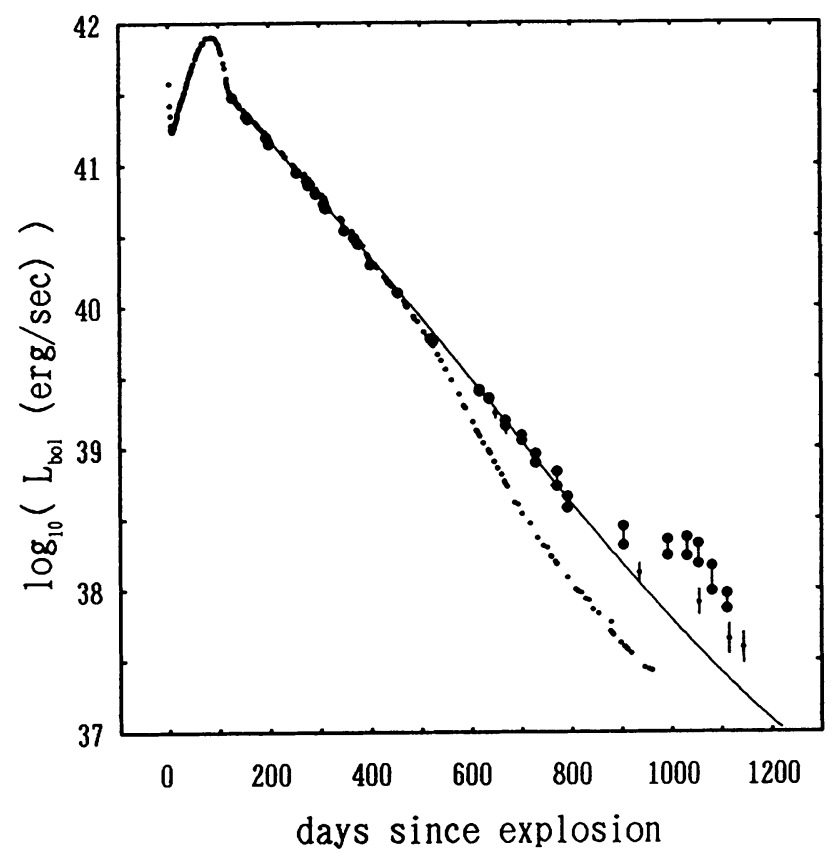

Fig. 3: The bolometric light curve due to the decays of ${ }^{56} \mathrm{Co}$ and ${ }^{57} \mathrm{Co}$ for $14 \mathrm{E} 1$ is compared with the light curve of SN 1987A observed with ESO (Bouchet et al. 1990), CTIO (Elias et al. 1990), and SAAO (Whitelock et al. 1989).

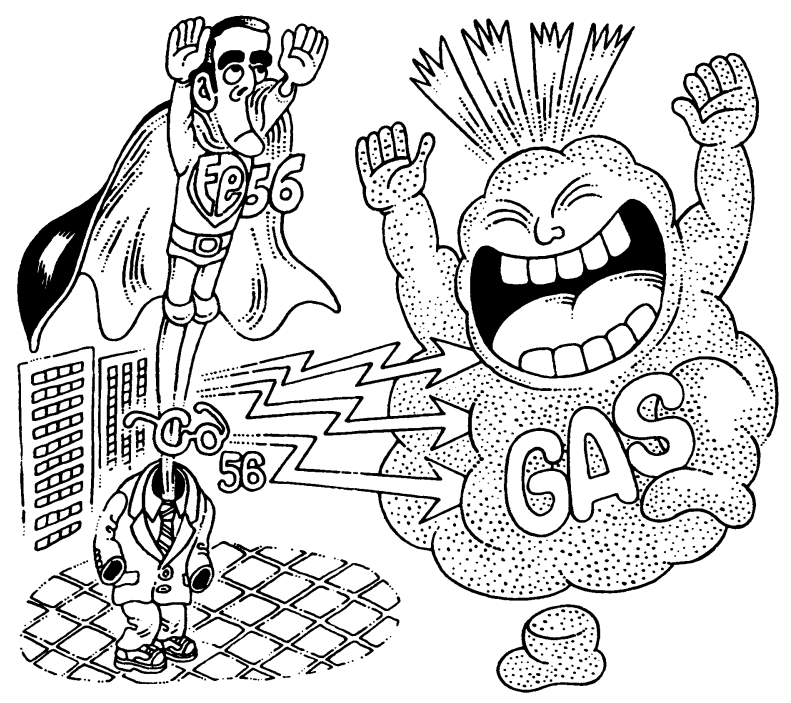

Fig. 4: The expanding supernova matter is excited by the decay of ${ }^{56} \mathrm{Co}$ into ${ }^{56} \mathrm{Fe}$ (H. Nomoto 1989). 
$120 \mathrm{~d}-400 \mathrm{~d}$ (Fig. 3) at a rate which coincides with the energy generation rate due to the Co-decay

$$
L=1.43 \times 10^{43} M_{\mathrm{Co}} / M_{\odot} e^{-t / 111.3 \mathrm{~d}} \quad \operatorname{ergs~s}^{-1},
$$

with $M_{\mathrm{Co}}=0.075 M_{\odot}$ (Catchpole et al. 1987; Suntzeff et al. 1988). The energy source that continually heats up the expanding star is certainly the decaying ${ }^{56} \mathrm{Co}$. The mass of ${ }^{56} \mathrm{Ni}$ thus determined as $\sim 0.075 M_{\odot}$ places the mass cut between the ejecta and the remaining neutron star at $1.60 M_{\odot}$.

The infrared spectra revealed the emission lines of heavy elements such as $\mathrm{Ne}$, $\mathrm{S}, \mathrm{Ar}, \mathrm{Ca}, \mathrm{Ni}, \mathrm{Co}$, and Fe (e.g., Danziger et al. 1990 for a review). These elements are certainly freshly synthesized elements as seen from their estimated masses. In particular, the observed time change of masses of $\mathrm{Co}$ and $\mathrm{Fe}$ is in good agreement with those resulting from decay of $\sim 0.07 M_{\odot}{ }^{56} \mathrm{Co}$ (e.g., Danziger et al. 1990). The mass of stable nickel $\left({ }^{58} \mathrm{Ni}\right.$ etc.) has been estimated to be $3-5 \times 10^{-3} M_{\odot}$ at day 400 (Danziger et al. 1990; Witteborn et al. 1989). This is in better agreement with the abundance given Nomoto et al. (1990a) which is the update of Hashimoto et al. (1989). Observed mass estimates of other elements are approximately consistent with Fig. 2.

\section{TYPE IB/IC SUPERNOVAE}

\subsection{Observations}

Figure 6 shows the observed bolometric curves of SNe Ia 1972E and 1981B (Graham 1987), SN Ib 1983N (Panagia 1987), and the approximate bolometric light curve of SN Ic 1987M constructed from flux-calibrated spectra (Filippenko et al. 1990; Nomoto et al. 1990c). In each case, the observed light curve has been shifted along the abscissa to match the corresponding theoretical curve. The peak bolometric luminosities assume $H_{0}=60 \mathrm{~km} \mathrm{~s}^{-1} \mathrm{Mpc}^{-1}$.

Wolf-Rayet stars with a wide range of masses have been proposed to be candidates for the progenitors of SNe Ib and Ic, since most of $\mathrm{SNe} \mathrm{Ib}$ are associated with star-forming regions (Wheeler and Levreault 1985; Begelman and Sarazin 1986; Gaskel et al. 1986; Uomoto 1986; Schlegel and Kirshner 1989; Schaeffer et al. 1987; Ensman and Woosley 1988; Nomoto et al. 1988a,b).

However, the previous Wolf-Rayet star models have some difficulties (1) in reproducing the light curves of typical SNe Ib which decline as fast as SNe Ia (Panagia 1987; Leibundgut 1988), and (2) in producing enough ${ }^{56} \mathrm{Ni}$ to attain the maximum luminosities of $\mathrm{SNe} \mathrm{Ib}$ in relatively low mass helium star models (Ensman and Woosley 1988). In particular, Figure 5 demonstrates two important features of SN Ic 1987M: (1) its brightness fell somewhat more rapidly than that of SNe Ia and SN Ib 1983N (also SN Ic 1983I reported by Tsvetkov 1985), and (2) maximum brightness 
of $1987 \mathrm{M}$ is only $0.5 \mathrm{mag}$ dimmer than in SNe Ia, and significantly brighter than in SN Ib $1983 \mathrm{~N}$ which was $\sim 1.5$ mag fainter than SNe Ia at maximum (Uomoto and Kirshner 1985; Branch 1986; Panagia 1987). These features are very difficult to understand with the previous massive helium star models.

Recently Shigeyama et al. (1990) have shown that the fast declines of the $\mathrm{SNe} \mathrm{Ib} / \mathrm{Ic}$ light curves are well reproduced if mixing of ${ }^{56} \mathrm{Ni}$ occurs in $3-5 M_{\odot}$ helium stars. Here we review nucleosynthesis and light curves of exploding helium stars to suggest that the helium stars of 3-5 $M_{\odot}$ (which form from stars with initial masses $M_{\mathrm{i}} \sim 12-18 M_{\odot}$ in binary systems) are the most likely progenitors of typical $\mathrm{SNe} \mathrm{Ib} / \mathrm{Ic}$.

\subsection{Nucleosynthesis and the Mass of ${ }^{56} \mathrm{Ni}$}

In the helium star models for $\mathrm{SNe} \mathrm{Ib}$, two scenarios are possible for the presupernova evolution. (1) A fairly massive single star lost its hydrogen-rich envelope in a wind. (2) A star in a close binary system becomes a helium star by Roche-lobe overflow.

Shigeyama et al. (1990) adopted the second scenario and performed hydrodynamical calculations of the explosion of the helium stars of masses $M_{\alpha}=3.3,4$, and $6 M_{\odot}$, which are presumed to form from the main-sequence stars of masses $M_{\mathrm{i}} \sim$ 13,15 , and $20 M_{\odot}$, respectively. These stars eventually undergo iron core collapse as in SNe II. A shock wave is then formed at the mass cut that divides the neutron star and the ejecta, propagating outward to explosively synthesize ${ }^{56} \mathrm{Ni}$ and other heavy elements as described in $§ 2.1$.

Since the mechanism that transforms collapse into explosion is unclear, the mass cut and explosion energy are not known. The adopted presupernova models (Nomoto and Hashimoto 1988) have the following important difference from the previous models, i.e., the iron core masses are as small as $1.18 M_{\odot}$ and $1.28 M_{\odot}$ for $M_{\alpha}=$ $3.3 M_{\odot}$ and $4 M_{\odot}$, respectively, significantly smaller than $1.4 M_{\odot}$ in the $6 M_{\odot}$ star, due to the larger effect of Coulomb interactions during the progenitor's evolution. Because of steep density gradient at the outer edge of the iron core, it is reasonable to assume that the neutron star mass $M_{\mathrm{NS}}$ is close to the iron core mass (Shigeyama et al. 1990). The final kinetic energy of explosion is assumed to be $E=1 \times 10^{51}$ erg.

As discussed in $\S 2.1$, materials are processed into nuclear statistical equilibrium (NSE) composition, mostly ${ }^{56} \mathrm{Ni}$, in a sphere of radius $\sim 3700\left(E / 10^{51} \mathrm{erg}\right)^{1 / 3} \mathrm{~km}$. This region contains a mass $M_{\mathrm{NSE}}\left(\sim 1.44-1.46 M_{\odot}\right.$ for $M_{\alpha}=3.3$ and $\left.4 M_{\odot}\right)$. Therefore the mass of ${ }^{56} \mathrm{Ni}$ is approximately given by $M_{\mathrm{NSE}}-M_{\mathrm{NS}}$. Distribution of the nucleosynthesis products for $M_{\alpha}=4 M_{\odot}$ is shown in Fig. 5 . The ${ }^{56} \mathrm{Ni}$ masses are 0.26 and $0.15 M_{\odot}$ for $M_{\alpha}=3.3$ and $4 M_{\odot}$, respectively, which are large enough to account for maximum brightness of SNe Ib/Ic. The oxygen masses are 0.21 and $0.43 M_{\odot}$ for $M_{\alpha}=3.3$ and $4 M_{\odot}$, respectively, and could be consistent with those 


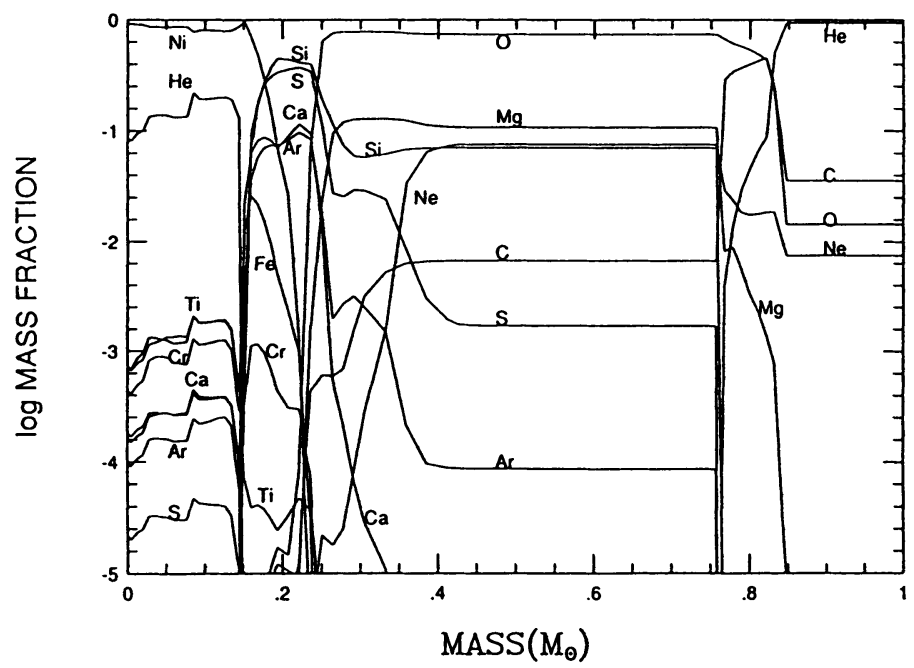

Fig. 5: Explosive nucleosynthesis in the $4 M_{\odot}$ helium star (Shigeyama et al. 1990). Composition of the innermost $1 M_{\odot}$ of the ejecta is shown. (The outermost 1.72 $M_{\odot}$ helium layer and the 1.28 $M_{\odot}$ neutron star are not included in the figure.) About $0.15 M_{\odot}{ }^{56} \mathrm{Ni}$ and $0.43 M_{\odot}$ oxygen are produced.

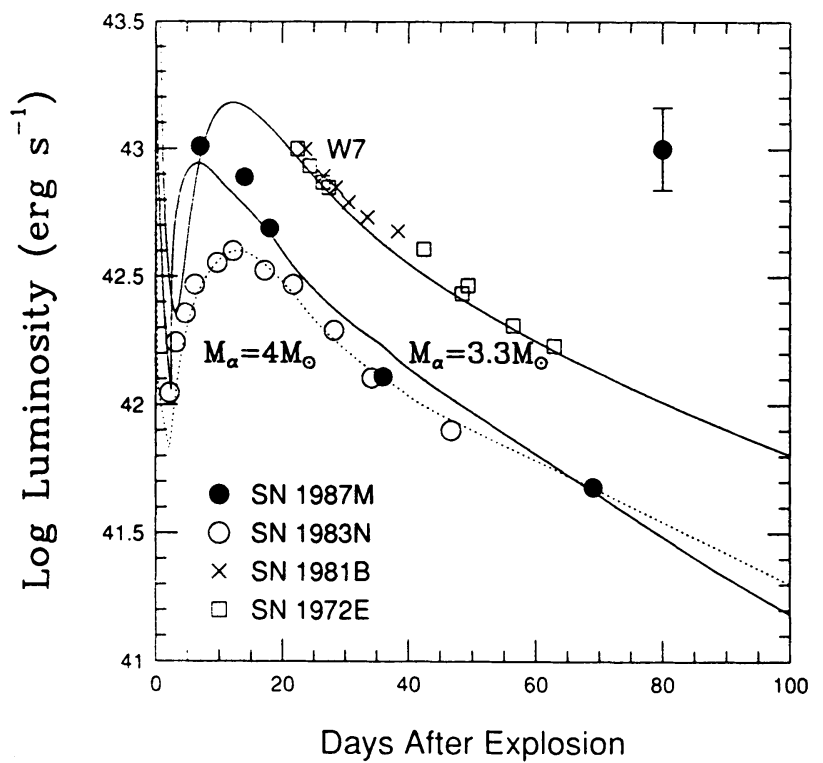

Fig. 6: Approximate bolometric light curve of SN Ic 1987M, and the bolometric light curves of SNe Ia 1972E and 1981B and of SN Ib 1983N. The predicted curves of the $3.3 M_{\odot}$ model for SN $1987 \mathrm{M}$, the $4 M_{\odot}$ model for SN Ib, and the W7 model for SN Ia are indicated by solid and dotted lines. The error bar illustrates the $2 \sigma$ photometric uncertainty in the SN 1987M points. 
inferred from the late time spectra of SNe Ib/Ic in view of the strong dependence of the oxygen mass on the temperature of the ejecta (e.g., Uomoto 1986).

\subsection{Light Curve Models}

Figure 6 shows the calculated bolometric light curves of the exploding helium star models with $M_{\alpha}=3.3 M_{\odot}$ for SN Ic and $4 M_{\odot}$ for SN Ib as well as the white dwarf model W7 for SNe Ia (§4). The amount of ${ }^{56} \mathrm{Ni}$ is $0.58 M_{\odot}(\mathrm{W} 7), 0.26 M_{\odot}$ (SN Ic), and $0.15 M_{\odot}$ (SN Ib). The helium star models assume uniformly mixed distribution of elements from the center through the layer at $0.2 M_{\odot}\left(0.3 M_{\odot}\right)$ beneath the surface for $M_{\alpha}=3.3 M_{\odot}\left(4 M_{\odot}\right)$. Such a mixing is due to Rayleigh-Taylor instability during the explosion as demonstrated by $2-\mathrm{D}$ hydrodynamical simulations (Hachisu et al. 1991).

The calculated bolometric light curves of helium stars are powered by the radioactive decays of ${ }^{56} \mathrm{Ni}$ and ${ }^{56} \mathrm{Co}$ as described in $\$ 2.2$. Peak luminosity is reached when the time scale of heat diffusion from the radioactive source becomes comparable to the expansion time scale. Maximum brightness is higher if the ${ }^{56} \mathrm{Ni}$ mass is larger and the date of maximum earlier. After the peak, the optical light curve declines at a rate that depends on how fast $\gamma$-rays from the radioactive decays escape from the star without being thermalized. Since the escape probability of $\gamma$-rays is determined by the column depth to the $\mathrm{Ni}-\mathrm{Co}$ layer, the optical light curve declines faster if the ejected mass is smaller and if ${ }^{56} \mathrm{Ni}$ is mixed closer to the surface.

Significant effects of mixing on the light curve has been noted in Shigeyama et al. (1990). For the unmixed cases, the calculated light curve tail declines much more slowly than that of $\mathrm{SN} 1983 \mathrm{~N}$ and $87 \mathrm{M}$ after $\sim$ day 40 . For the mixed cases shown in Fig. 6, the light curve shape is different from the unmixed cases as follows:

(1) The maximum luminosity is reached $\sim 10$ days earlier and thus being higher than in the unmixed cases because of the earlier radioactive heating of the surface layers.

(2) The decline of the tail is much faster than the unmixed cases.

Figure 6 shows that the bolometric light curve of $M_{\alpha}=4 M_{\odot}$ is in excellent agreement with SN Ib $1983 \mathrm{~N}$ from the pre-maximum through day 50 . Other wellobserved SNe Ib generally have early-time visual and bolometric light curves whose shapes are nearly identical to those of SNe Ia (Porter and Filippenko 1987; Leibundgut 1988).

Compared with the $4 M_{\odot}$ model for $\mathrm{SNe} \mathrm{Ib}$, the $3.3 M_{\odot}$ model has several attractive features for SN 1987M. First, maximum brightness is higher by more than a factor of two because of the larger ${ }^{56} \mathrm{Ni}$ mass and the earlier date of maximum brightness. Second, decline in the tail is noticeably faster due to the more extensive mixing and smaller ejected mass. Compared with the W7 model for SNe Ia, the $3.3 M_{\odot}$ model gives a lower maximum brightness (by about $0.6 \mathrm{mag}$ ) and a faster 
decline, just as observed in SN 1987M.

It is interesting to compare $\mathrm{SNe} \mathrm{Ib} / \mathrm{Ic}$ and $\mathrm{SN} 1987 \mathrm{~K}$ whose spectral classification changed from Type II to Type Ib/Ic as it aged, thereby being called as $\mathrm{SNe}$ IIb (Filippenko 1988). The decline of the SN 1987K light curve is as fast as SNe Ia (and thus SNe Ic) (Turatto et al. 1990). The early time spectra of SN 1987K are very similar to SNe Ic $(1983 \mathrm{~V}$ and $87 \mathrm{M})$ and, conversely, hydrogen feature has been identified in the early time spectrum of SN Ic 1987M (Jeffery et al. 1990). This strongly suggests that the difference in the spectral feature between SNe Ic and Ib is due to the presence of a thin envelope of hydrogen in SNe Ic immediately prior to the explosion. More hydrogen can be left on the smaller mass helium stars after mass exchange and wind-type mass loss (Yamaoka and Nomoto 1990).

\section{TYPE IA SUPERNOVAE}

\subsection{Evolution of White Dwarf Progenitors}

Accreting white dwarfs in close binary systems have been considered to be the most promising candidates of the Type Ia supernova progenitors. There are several possible evolutionary scenarios from the beginning of accretion through the thermonuclear explosion. Initially the close binary system consists of two intermediate mass stars. As a result of Roche lobe overflow, the primary star of this system becomes a white dwarf composed of carbon and oxygen $(\mathrm{C}+\mathrm{O})$. When the secondary star evolves, it begins to transfer hydrogen-rich matter over to the white dwarf. If the accretion rate is larger than a certain rate, the binary enters a common envelope phase. The common envelope evolution may lead to the formation of (1) a white dwarf - helium main-sequence pair (Tornambe and Matteucci 1986), (2) a C+O white dwarf pair (Iben and Tutukov 1984; Webbink 1984), and (3) a common envelope in which the $\mathrm{C}+\mathrm{O}$ white dwarf burns hydrogen steadily (Hachisu et al. 1989). Further evolution of the system leads to an accretion of (1) helium, (2) $\mathrm{C}+\mathrm{O}$, and (3) hydrogen onto the $\mathrm{C}+\mathrm{O}$ white dwarf.

The fate of such accreting white dwarfs depends primarily on the mass accretion rate and, hence, there are several possible mechanisms of explosion (Nomoto and Kondo 1991). Among them the most plausible model for SN Ia events is the carbon deflagration of a $\mathrm{C}+\mathrm{O}$ white dwarf. This takes place for accretion at intermediate rates $\left(2.7 \times 10^{-6} \quad M_{\odot} \mathrm{yr}^{-1}>\dot{M}>1 \times 10^{-8} \quad M_{\odot} \mathrm{yr}^{-1}\right)$. Here we describe the carbon deflagration model W7 calculated by Nomoto et al. (1984) and Thielemann et al. (1986) as a standard model (see Woosley and Weaver 1986 and Wheeler and Harkness 1990 for reviews and references therein).

\subsection{Carbon Deflagration}

When the central density of the $\mathrm{C}+\mathrm{O}$ white dwarf becomes as high as $2 \times 10^{9} \mathrm{~g}$ 


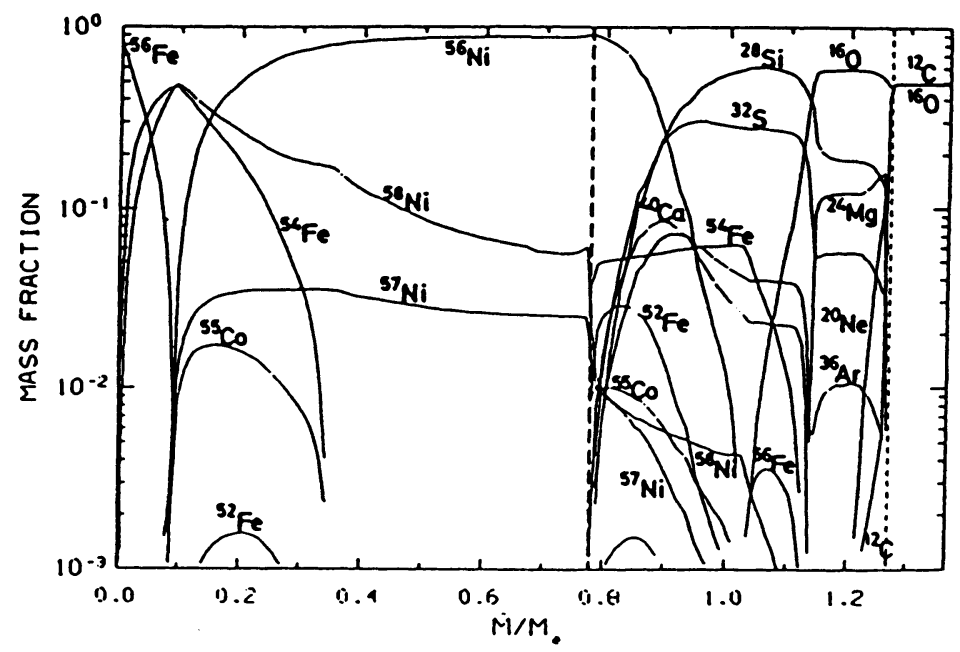

Fig. 7: Composition of a carbon deflagration model for Type Ia supernovae as a function of interior mass. The white dwarf undergoes incineration into iron-peak elements (mostly ${ }^{56} \mathrm{Ni}$ ) at $M_{r}<0.7 M_{\odot}$, and partial explosive burning in the intermediate region at $0.7 M_{\odot}<M_{r}<1.3 M_{\odot}$; in the outermost layer, carbon and oxygen remain unburned.

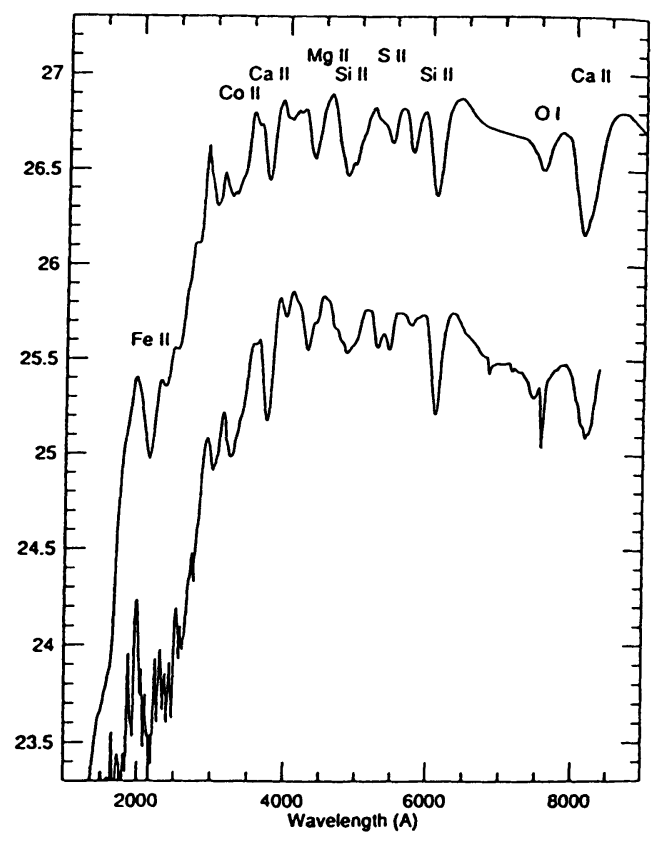

Fig. 8: The maximum light spectrum of SN 1981B is compared with a synthetic spectrum for the carbon deflagration model shown in Fig. 7. 
$\mathrm{cm}^{-3}$, carbon burning is ignited at the center. It is so explosive as to incinerate the material into iron-peak elements; the central temperature reaches $\sim 10^{10} \mathrm{~K}$. The resulting shock wave is not strong enough to ignite carbon in the adjacent layer; in other words, a detonation wave that propagates at a supersonic speed does not form. Instead the interface between the burned and unburned layers becomes convectively unstable. As a result of mixing with the hot material, fresh carbon is ignited. In this way, an explosive carbon burning front propagates outward propagates at a subsonic speed (a convective deflagration wave). In model $\mathrm{W} 7$, the time-dependent mixing length theory with mixing length $\ell=0.7$ times a pressure scale height is adopted. It takes about $1 \mathrm{sec}$ for the front to reach the surface region, which is significantly slower than the supersonic detonation wave. Hence the white dwarf expands during the propagation of the deflagration wave.

Behind the deflagration wave, the material undergoes explosive nuclear burning of silicon, oxygen, neon, and carbon depending on the peak temperatures $T(\$ 2.2)$. In the inner layer, $T$ is high enough to incinerate the material into mostly ${ }^{56} \mathrm{Ni}$. When the deflagration wave arrives at the outer layers, the density it encounters has already decreased due to the expansion of the white dwarf. At such low densities, $T$ is too low to complete silicon burning and thus only $\mathrm{Ca}, \mathrm{Ar}, \mathrm{S}$, and $\mathrm{Si}$ are produced. In the intermediate layers, explosive burning of carbon and neon synthesizes $\mathrm{S}, \mathrm{Si}$, and $\mathrm{Mg}$. In the outermost layers, the deflagration wave dies and $\mathrm{C}+\mathrm{O}$ remains unburned. The nucleosynthesis products after freeze-out are seen in the tables in Nomoto et al. (1984) and Thielemann et al. (1986) and the composition structure is shown in Figure 7.

In model $\mathrm{W} 7$, the amount of ${ }^{56} \mathrm{Ni}$ produced is $M_{\mathrm{Ni}}=0.58 M_{\odot}$, and the explosion energy is $E=1.3 \times 10^{51} \mathrm{erg}$. The nuclear energy release is large enough to disrupt the white dwarf completely and no neutron star is left behind.

\subsection{Spectra and Light Curves}

Since SNe Ia do not have a thick hydrogen/helium-rich envelope, newly synthesized elements during the explosion can be observed in the spectra; this enable us to diagnose the internal hydrodynamics and nucleosynthesis in SNe Ia.

Synthetic spectra are calculated based on the abundance distribution and expansion velocities of the standard model and found to be in excellent agreement with the observed optical spectrum of SN 1981b as seen in Fig. 8 (IIarkness and Wheeler 1990). The material velocity at the photosphere near maximum light is $\sim 10,000$ $\mathrm{km} \mathrm{s}^{-1}$ and the spectral features are identified as P-Cygni profiles of $\mathrm{Fe}, \mathrm{Ca}, \mathrm{S}, \mathrm{Si}$, $\mathrm{Mg}$, O (Branch et al. 1985). The agreement implies that both explosion energy and nucleosynthesis in the carbon deflagration model are consistent with SN Ia.

At late times, the spectra are dominated by the emission lines of $\mathrm{Fe}$ and $\mathrm{Co}$. The outer layers are transparent and the inner Ni-Co-Fe core is exposed. Synthetic 


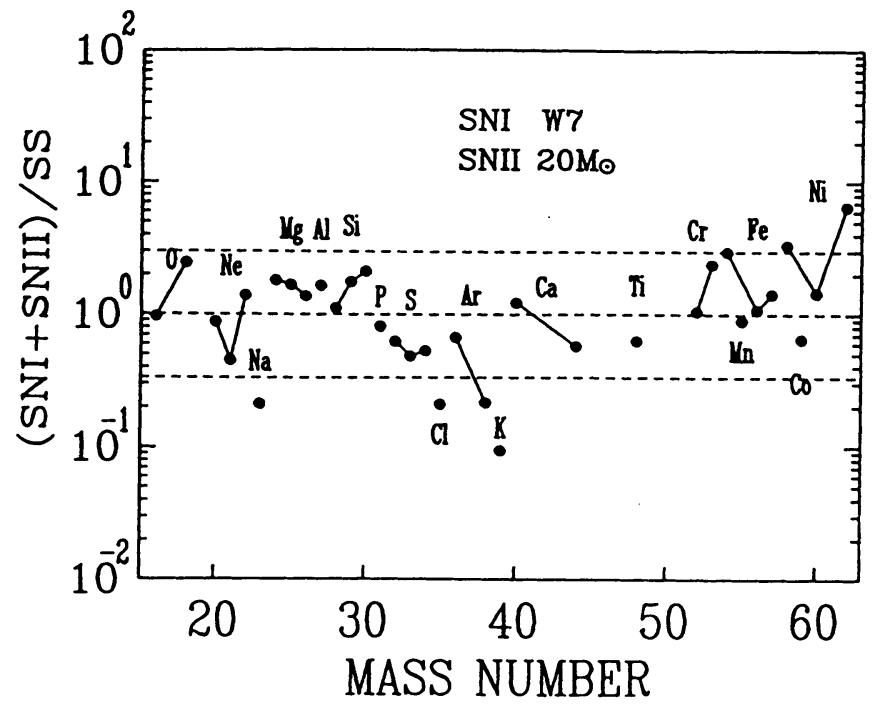

Fig. 9: The combined nucleosynthesis products from SN Ia (W7) and SN II ( $20 M_{\odot}$ star). The SN Ia/SN II ratio of $0.17 / 0.83$ is obtained by minimizing the deviation from the solar abundances.

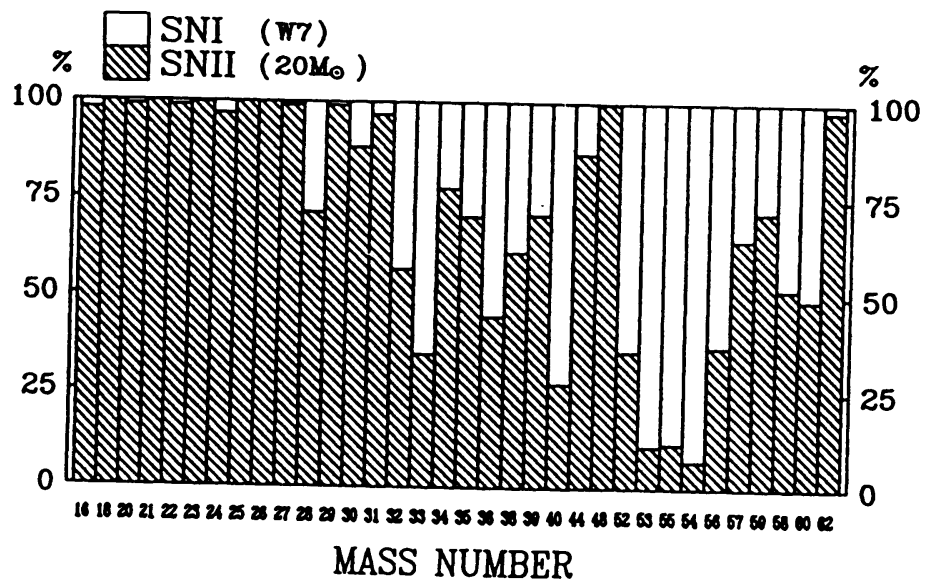

Decomposition into tro components for each isotope in the Solar System.

Fig. 10: The relative contributions of SN Ia and SN II for each species in best reproducing the solar abundances. 
spectra of emission lines of [Fe II] and [Co I] agree quite well with the spectra observed at such phase (Axelrod 1980).

The explosion energy goes into the kinetic energy of expansion, and without a late time energy source the exploding white dwarf could not be bright. However, radioactive decays of ${ }^{56} \mathrm{Ni}$ and ${ }^{56} \mathrm{Co}$ heat up the expanding materials and produce the optical light. The light curve declines faster than the ${ }^{56} \mathrm{Co}$ decay because of the increasing transparency of the ejecta to $\gamma$-rays. The calculated light curve of W7 is in good agreement with the observed bolometric light curves of SN 1972E and SN $1981 B$ as seen later in Fig. 6.

\section{SUPERNOVAE AND CHEMICAL EVOLUTION OF GALAXIES}

Supernovae are the sites of heavy element production in galaxies. Because of the short lifetimes of their progenitors, SNe II and SNe Ib/Ic contribute the heavy element production in the early chemical evolution of galaxies. SNe Ia, on the other hand, enhances mostly iron in the late phase galactic evolution. To quantitatively demonstrate the relative nucleosynthesis roles of SNe Ia and SNe II, Nomoto et al. (1990b; also Yanagida et al. 1990) sum up the nucleosynthesis products as [r $\left.X_{\mathrm{i}, \mathrm{Ia}}+(1-r) X_{\mathrm{i}, \mathrm{II}}\right]$ and determine $r$ by minimizing the deviation from the solar abundances (Anders and Grevesse 1989). For SNe II, we simply assume that SNe II yield is represented by the $20 M_{\odot}$ product ( $\$ 2$; Hashimoto et al. 1989; Thielemann et al. 1990). For SN Ia, the W7 model is adopted ( $\$ 4$; Nomoto et al. 1984; Thielemann et al. 1986). The best fit to the solar abundances is obtained for $r=0.17$ as shown in Fig. 9, where the deviation from the solar is at most a factor of 3. The contribution of SNe Ia and SNe II for each species is shown in Fig. 10; for example, $\sim 63$ percent of $\mathrm{Fe}$ originate from SN Ia. The obtained ratio may be consistent with that observed estimate by Evans et al. (1989) though a large uncertainty is involved.

Nucleosynthesis in SNe II can be compared with the abundance of metal-poor stars. For this purpose, we use the relation between the supernova types and progenitor's masses in Figure 1 as a working hypothesis, and examine the relative contributions of different types of supernovae. Stars more massive than initially $\sim 12$ $M_{\odot}$ end up as SNe II-P if single stars and as SNe Ib/Ic in close binary systems. Figure 1 predicts that, for $12 M_{\odot} \lesssim M_{\mathrm{i}} \lesssim 20 M_{\odot}$, lower mass stars produce larger amounts of ${ }^{56} \mathrm{Ni}$ than do higher mass stars. On the other hand, the oxygen mass produced from these massive stars is larger for more massive stars, if no mass loss is assumed. However, if the very massive stars undergo significant mass loss to become a relatively small mass Wolf-Rayet stars, their heavy element production is small. Therefore oxygen mass integrated over initial mass function (IMF) depends mainly on the upper mass limit of stars that do not evolve into Wolf-Rayet stars.

Figure 11 shows the evolutionary change in $[\mathrm{O} / \mathrm{Fe}]=\log _{10}\left[(\mathrm{O} / \mathrm{Fe}) /(\mathrm{O} / \mathrm{Fe})_{\odot}\right]$ as a function of metallicity $[\mathrm{Fe} / \mathrm{H}]$. (Observed points are taken from Andersen et 


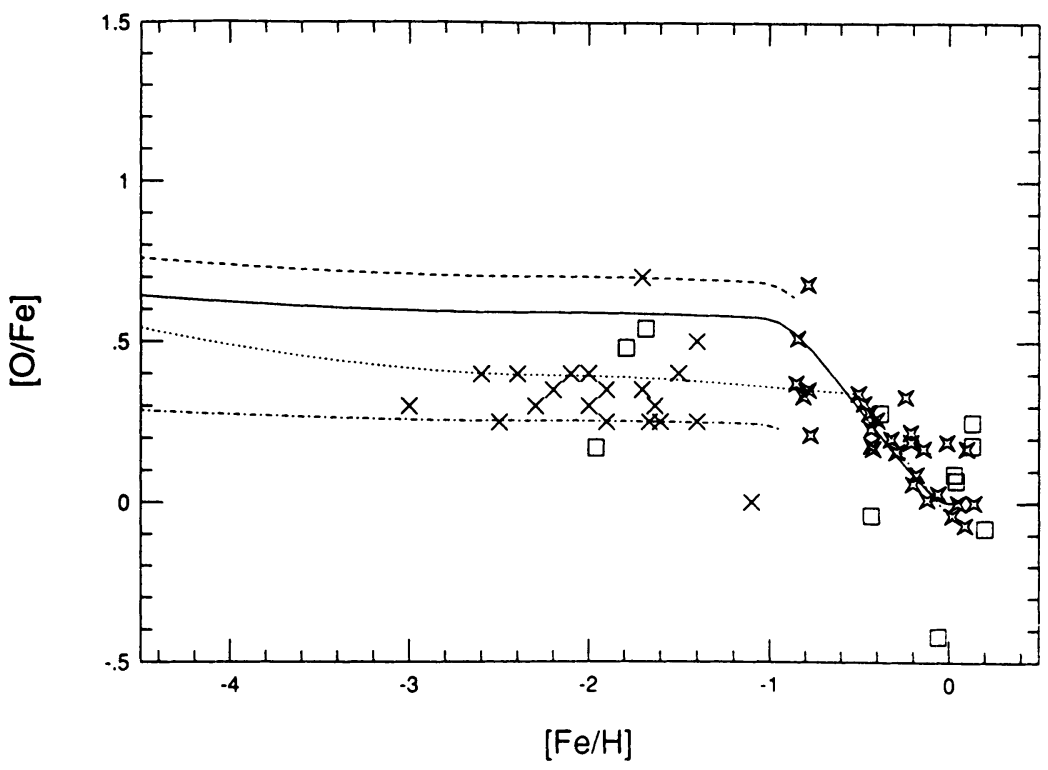

Fig. 11: The $[\mathrm{O} / \mathrm{Fe}]$ ratio against metallicity $[\mathrm{Fe} / \mathrm{H}]$ observed in low metallicity stars and calculated with four different theoretical models (see text).

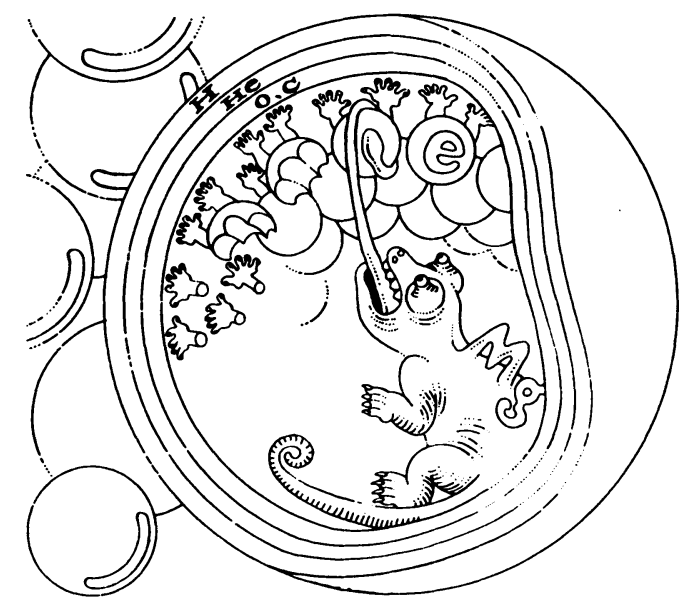

Fig. 12: Collapse of an $\mathrm{O}+\mathrm{Ne}+\mathrm{Mg}$ core is induced by electron capture (H. Nomoto 1989). 
al. 1988, Barbuy 1988, and Gratton and Ortolani 1986.) The decrease in $[\mathrm{O} / \mathrm{Fe}]$ at $[\mathrm{Fe} / \mathrm{H}] Z^{-1}$ is due to the iron production from SNe Ia whose lifetime is assumed to be $\sim 1$ Gyr. For massive stars, integration of the yield over $12-100 M_{\odot}$ (case A) and $12-35 M_{\odot}$ (case B) is made with Salpeter's IMF, where the Fe mass produced from stars more massive than $20 M_{\odot}$ is assumed to be $0.07 M_{\odot}$. The resulting integrated yields of SNe II give $[\mathrm{O} / \mathrm{Fe}]=0.70$ (case A: dashed line) and 0.26 (case $\mathrm{B}$ : dash-dotted line) at metallicity of $[\mathrm{Fe} / \mathrm{H}] \lesssim-1$ (solid line). The dotted line (case $\mathrm{C}$ ) is the integration over $12-100 M_{\odot}$ but oxygen yield is saturated at constant value for $40-100 M_{\odot}$; the resulting $[\mathrm{O} / \mathrm{Fe}]$ is marginally consistent with the observed $[\mathrm{O} / \mathrm{Fe}]=0.41 \pm 0.15$ averaged over low metallicity stars of $[\mathrm{Fe} / \mathrm{H}] \lesssim-1$ in the solar neighborhood (Gratton 1990). These cases demonstrate that oxygen production is largely contributed by $25-40 M_{\odot}$ stars.

In Fig. 1, SNe II-BL (bright linear, SN 1979C; D. Branch, private communication) and SNe II-L are tentatively assumed to be the explosions of AGB stars having degenerate $\mathrm{C}+\mathrm{O}$ cores (carbon deflagration) and $\mathrm{O}+\mathrm{Ne}+\mathrm{Mg}$ cores (electron capture collapse; Fig. 12), respectively (Swartz et al. 1990). SNe II-L and II-BL do not show a plateau, which suggests that their progenitors have hydrogen-rich envelope less than $\sim 1 M_{\odot}$. This is consistent with the AGB star models since they are likely to have lost most of their hydrogen-rich envelopes at the explosion.

The above models imply that the yields of SNe II-BL are almost identical to those of SN Ia, while SNe II-L may not produce much heavy elements. The solid line in Fig. 11 includes the production of $0.58 M_{\odot}$ Fe from SNe II-BL assuming 7.6 $M_{\odot} \leq M_{\mathrm{i}} \leq 8 M_{\odot}$ for their mass range. To be consistent with $[\mathrm{O} / \mathrm{Fe}] \gtrsim 0.26$ for metal-poor stars, the mass range for SNe II-BL must be as narrow as $7.2 M_{\odot} \lesssim M_{\mathrm{i}}$ $\lesssim 8 M_{\odot}$. This is consistent with the fact that only one (or two) example of SN II-BL (i.e., SN $1979 \mathrm{C}$ and possibly $1980 \mathrm{~K}$ ) have been observed despite their brightness. If we take 7.6 $M_{\odot}-8 M_{\odot}$ for $M_{\mathrm{i}}$ of SNe II-BL, the Fe mass from SNe II-L is limited to $<0.1 M_{\odot}$.

We would like to thank S. Kumagai, H. Yamaoka, M. Hashimoto, F.-K. Thielemann, A.V. Filippenko, D. Branch, S. Yanagida, and S. Hayakawa for collaborative work on the subjects discussed in this paper. This work has been supported in part by the grant-in-Aid for Scientific Research (01540216, 01790169, 02234202, 02302024) of the Ministry of Education, Science, and Culture in Japan, and by the Japan-U.S. Cooperative Science Program (EPAR-071/88-15999) operated by the JSPS and the NSF.

\section{REFERENCES}

Anders, E., and Grevesse, N. 1989, Geochim. Cosmochim. Acta, 53, 197. 
Andersen, J., Edvardsson, B., Gustafsson, B., and Nissen, P.E. 1988, in IAU Symp. 132, The Impact of Very High $S / N$ Spectroscopy on Stellar Physics, ed. G.

Cayrel, de Strobel, M. Spite (Dordrecht: Kluwer), p.441.

Arnett, W.D. 1988, Ap. J., 334, 500.

Arnett, W.D., Bahcall, J.N., Kirshner, R.P., and Woosley, S.E. 1989, Ann. Rev. Astr. Ap., 27, 629.

Axelrod, T. 1980, in Type I Supernovae, ed. J.C. Wheeler (Univ. of Texas), p. 80.

Barbuy, B. 1988, Astr. Ap., 191, 121.

Begelman, M.C., and Sarazin, C.L. 1986, Ap. J. (Letters), 302, L59.

Bouchet, P., Danziger, I.J., and Lucy, L. 1990, in SN 1987A and Other Supernovae, ed. I.J. Danziger (Garching: ESO), in press.

Branch, D. 1986, Ap. J. (Letters), 300, L51.

Branch, D., Doggett, J.B., Nomoto, K., and Thielemann, F.-K. 1985, Ap. J., 294, 619.

Catchpole, R. et al. 1987, M. N. R. A. S., 229, 15p.

Danziger, I.J., Lucy, L.B., and Bouchet, 1990, in Supernovae, ed. S.E. Woosley (Springer-Verlag), in press.

Elias, J.H. et al. 1990, in SN $1987 A$ and Other Supernovae, ed. I.J. Danziger (Garching: ESO), in press.

Evans, R., van den Bergh, S., McClure, R.D. 1989, Ap. J. 345, 752

Ensman, L., and Woosley, S.E. 1988, Ap. J., 333, 754.

Filippenko, A.V. 1988, A. J., 96, 1941.

Filippenko, A.V., Porter, A.C., and Sargent, W.L.W. 1990, A. J., 100, 1575.

Gaskell, C.M., Cappellaro, E., Dinerstein, H.L., Garnett, D.R., Harkness, R.P., and Wheeler, J.C. 1986, Ap. J. (Letters), 306, L77.

Graham, J.R. 1987, Ap. J., 315, 588.

Gratton, R.G. 1990, this volume.

Gratton, R.G., and Ortolani, S. 1986, Astr. Ap., 169, 201.

Hachisu, I., Kato, M., and Saio, H. 1989, Ap. J. (Letters), 342, L19.

Hachisu, I., Matsuda, T., Nomoto, K., and Shigeyama, T. 1991, Ap. J. (Letters), in press.

Harkness, R.P., and Wheeler, J.C. 1990, in Supernovae, ed. A. Petschek (SpringerVerlag), p. 1.

Hashimoto, M., Nomoto, K., and Shigeyama, T. 1988, Astr. Ap., 210, L5.

Iben, I.Jr., and Tutukov, A. 1984, Ap. J. Suppl., 55, 335.

Jeffery, D., Branch, D., Filippenko, A.V., and Nomoto, K. 1990, preprint.

Kumagai, S., Shigeyama, T., Nomoto, K., Itoh, M., Nishimura, J., and Tsuruta, S. 1989, Ap. J., 345, 412.

Leibundgut, B. 1988, Ph.D. thesis, Universität Basel.

Nomoto, H. 1989, Exploring SN 1987A (in Japanese), (Tokyo: Kodansha).

Nomoto, K., Filippenko, A.V., and Shigeyama, T. 1990c, Astr. Ap., in press.

Nomoto, K., and Hashimoto, M. 1988, Physics Reports, 163, 13.

Nomoto, K., and Kondo, Y. 1991, Ap. J. (Letters), in press. 
Nomoto, K., Shigeyama, T., and Hashimoto, M. 1988a, in IAU Colloquium 108, Atmospheric Diagnostics of Stellar Evolution, ed. K. Nomoto, Lecture Notes in Physics, 305, 319.

Nomoto, K., Shigeyama, T., Kumagai, S., and Hashimoto, M. 1988b, in Proc. Astr. Soc. Australia, 7, 490.

Nomoto, K., Shigeyama, T., Kumagai, S., and Yamaoka, H. 1990a, in Supernovae, ed. S.E. Woosley (Springer-Verlag), in press.

Nomoto, K., Shigeyama, T., Yanagita, S., Hayakawa, S., and Yasuda, K. 1990b, in Chemical and Dynamical Evolution of Galaxies, eds. F. Ferrini, J. Franco, and F. Matteucci (Pisa: Giondini), in press.

Nomoto, K., Thielemann, F.-K., and Yokoi, K. 1984, Ap. J., 286, 644.

Panagia, N. 1987, in High Energy Phenomena Around Collapsed Stars, ed. F. Pacini (D. Reidel), p. 33.

Porter, A.C., and Filippenko, A.V. 1987, Astr. J. 93, 1372

Schaeffer, R., Casse, M., and Cahen, S. 1987, Ap. J., 316, L31.

Schlegel, E.M., and Kirshner, R.P. 1989, A. J., 98, 577.

Shigeyama, T., and Nomoto, K. 1990, Ap. J., 360, 242.

Shigeyama, T., Nomoto, K., and Hashimoto, M. 1988, Astr. Ap., $196,141$.

Shigeyama, T., Nomoto, K., Tsujimoto, T., and Hashimoto, M. 1990, Ap. J. (Letters), 361, L23.

Suntzeff, N.B., Hamuy, M., Martin, G., Gomez, A., and Gonzalez, R. 1988, A. J., 96, 1864.

Swartz, D.A., Wheeler, J.C., and Harkness, R.P., 1990, Ap. J., submitted.

Thielemann, F.-K., Hashimoto, M., and Nomoto, K. 1990, Ap. J., 349, 222.

Thielemann, F.-K., Nomoto, K., and Yokoi, K. 1986, Astr. Ap., 158, 17.

Tornambe, A., and Matteucci, F. 1986, M. N. R. A. S., 223, 69.

Tsvetkov, D.Yu. 1985, Sov. Astr., 29, 211.

Turatto, M., Cappellaro, E., Barbon, R., Della Valle, M., Ortolani, S., and Rosino, L. $1990, A . J$. , in press.

Uomoto, A. 1986, Ap. J. (Letters), 310, L35.

Uomoto, A., and Kirshner, R.P. 1985, Astr. Ap., 149, L7.

Webbink, R. 1984, Ap. J., 277, 355.

Wheeler, J.C., and Levreault, R. 1985, Ap. J. (Letters), 294, L17.

Wheeler, J.C., and Harkness, R. 1990, Phys. Rep., in press.

Whitelock, C. et al. 1989, M. N. R. A. S., 240, 7p.

Witteborn, F.C., Bregman, J.D., Wooden, D.H., Pinto, P.A., Rank, D.M., Woosley, S.E., and Cohen, M. 1989, Ap. J. (Letters), 338, L9.

Woosley, S.E. 1988, Ap. J., 330, 218.

Woosley, S.E., and Weaver, T.A. 1986, Ann. Rev. Astr. Ap., 24, 205.

Yamaoka, H., and Nomoto, K. 1990, in SN $1987 A$ and Other Supernovae, ed. I.J. Danziger (Garching: ESO), in press.

Yanagida, S., Nomoto, K., and Hayakawa, S. 1990, in Proceedings of the 21st International Cosmic Ray Conference (Adelaide), 4, 44. 